\title{
A prospective study to evaluate the role of curcumin in treatment of locally advanced carcinoma of cervix
}

\begin{abstract}
Objectives: The present study is conducted to determine the role of curcumin alongwith chemoradiation in the management of locally advanced carcinoma cervix in terms of toxicity, tumor response and survival rates.

Methods: Patients were enrolled into study from Jan 2012 to Jan 2014. The patients were randomized into two groups (group I and group II) of 30 patients each. All patients were planned for external beam radiotherapy (EBRT) to total dose of $50 \mathrm{~Gy} / 25$ fractions $/ 5$ weeks with concomitant cisplatin $40 \mathrm{mg} / \mathrm{m} 2$ IV weekly. HDR brachytherapy with 3 fractions of $6 \mathrm{~Gy}$ each was given weekly after completion of EBRT. Curcumin (4gm/day) was given orally to group I patients from the start of EBRT till the completion of brachytherapy.

Results: The baseline patients' characteristics were matched in the two groups. The median follow up was of two years. After the completion of treatment, complete tumor response was seen in $70 \%$ and $66.67 \%$ patients in group I and II respectively. Overall survival and disease free survival rates were $93.3 \%, 76.7 \%$ in group I and $90 \%$, $66.7 \%$ in group II respectively. The findings were not statistically significant. Grade 3 skin reactions were seen in $3.33 \%$ and $20 \%$ of the patients in group I and group II respectively. Acute mucosal toxicity of grade 2 was seen in $23.3 \%$ and $50 \%$ of patients in group I and II respectively.
\end{abstract}

Conclusion: The significant difference was found in occurrence of severe acute radiation toxicity to skin and mucosa with comparable survival rates in the two groups, i.e. with and without curcumin.

Keywords: curcmin, cervix, carcinoma, chemoradiation
Volume 5 Issue 4 - 2018

\author{
Nupur Bansal,' Ashok Kr Chauhan,' \\ Paramjeet Kaur,' Atreyee Basu, ${ }^{2}$ Anil \\ Khurana,' Yashpal Verma,' Meenakshi B \\ Chauhan $^{3}$ \\ 'Department of Radiotherapy, Pt. B.D. Sharma PGIMS, India \\ ${ }^{2}$ Biochemistry, India \\ ${ }^{3}$ Department of Obstetrics \& Gynaecology, Pt. B.D. Sharma \\ PGIMS, India
}

Correspondence: Nupur Bansal, Department of Radiotherapy, Pt BDS PGIMS, Rohtak, Haryana, India, Tel +9I-8053442667; Email drnupurbansal@gmail.com

Received: May 15, 2018 | Published: July 05, 2018
Abbreviations: CCRT, concurrent chemoradiotherapy; NF$\kappa \mathrm{B}$, nuclear factor-kappa B; STAT3, signal transducer and activator of transcription 3; NRF2, nuclear factor E2-related factor-2; $\mathrm{Hb}$, hemoglobin; TLC, total leucocyte count; EBRT, external beam radiotherapy; ICBT, intracavitary brachytherapy; WHO, world health organization; GIT, gastrointestinal tract; GUT, genitourinary tract; RTOG, radiation therapy oncology group; SWOG, south west oncology group, MGUS, monoclonal gammopathy of undetermined significance; RDS, radiation dermatitis severity

\section{Introduction}

Cervical cancer is the most frequent gynaecological cancer and the second leading cause of cancer-related deaths among women worldwide. ${ }^{1}$ Concurrent chemoradiotherapy (CCRT) with cisplatin based chemotherapy has been the standard line of treatment for locally advanced carcinoma of cervix. The randomized trials have shown that the use of concurrent chemoradiation results in a $30 \%$ to $50 \%$ decrease in the risk of death as compared to radiotherapy alone. Also, $6 \%$ improvement in 5 year survival was seen when compared with radiotherapy alone..$^{2-7}$ Although, it is well tolerated, acute and long term side effects have been seen. The attempts have been carried out to decrease toxicity of chemoradiation as well as to improve the outcome at the same time. Administration of curcumin in patients is supposed to kill the tumor cells effectively by enhancing the effect of radiation and, at the same time, protect normal cells against the harmful effects of radiation.

Curcumin is a product obtained from plant Curcuma longa. It has radioprotective and radiosensitising properties and thus, may be helpful in decreasing the toxicity and increasing the efficacy profile of the treatment. It acts as a chemosensitizer and radiosensitizer for tumors by downregulating various growth regulatory pathways and specific genetic targets including genes for Nuclear factor-kappa $\mathrm{B}(\mathrm{NF}-\mathrm{\kappa B})$, Cyclooxygenase-2, Signal transducer and activator of transcription 3 (STAT3), multidrug-resistance proteins, antiapoptotic proteins and growth factor receptors. ${ }^{8}$ Curcumin can induce the expression of some antioxidant enzymes and activation of Nuclear factor E2-related factor-2 (NRF2). This mechanism lead to the protective effect of curcumin and has shown to protect normal organs from chemotherapy and radiotherapy induced toxicity, such as liver, kidney, oral mucosa and heart. ${ }^{8}$

Before we started this study, there was no published study of its kind in the literature. The current study was designed to see if the addition of curcumin to standard treatment of locally advanced carcinoma of uterine cervix could improve the tumor control and reduce the toxicity profile in the patients.

\section{Material and methods}

Patients with histopathologically proven locally advanced carcinoma of cervix, who were treated from January 2012 to 
January 2014 were included in the study. All of the patients were staged according to International Federation of Gynecologists and Obstetricians (FIGO) staging system, 2010. All patients had the following characteristics: stage IIB to IVA, Karnofsky Performance Status $\geq 70$, normal renal function tests, liver function tests and complete hemogram. Their blood investigations were as per the eligibility criteria with Hemoglobin $(\mathrm{Hb})>8 \mathrm{gm} / \mathrm{dL}$, Total Leucocyte Count (TLC) $>4000 / \mathrm{cmm}$, platelet count $>100,000 / \mathrm{cmm}$, blood urea $<40 \mathrm{mg} / \mathrm{dL}$, serum creatinine $<1.5 \mathrm{mg} / \mathrm{dL}, \mathrm{SGOT}<35 \mathrm{IU} / \mathrm{L}$ and SGPT $<40$ IU/L. Patients with distant metastases, prior chemotherapy, surgery or radiation for the disease, associated co-morbid medical condition, history of prior malignancy were excluded from the study. Ethical clearance was obtained from institutional ethical committee. Informed written consent was obtained from all participants before the commencement of study.

\section{Radiation techniques}

External beam radiotherapy (EBRT) by Cobalt-60 unit was used to treat the whole pelvis. A total dose of 50Gy was delivered with the daily fraction of $2 \mathrm{~Gy}$, five days per week. The radiation was delivered by Cobalt-60 unit. Intracavitary brachytherapy (ICBT) was started after the EBRT and three sessions of 6Gy, one session per week for three consecutive weeks were given by Ir-192 microselectron HDR remote controlled afterloading brachytherapy machine. All patients were treated with concurrent chemoradiotherapy. Patients were prospectively randomized into treatment schedules:

Group I: Cisplatin+Curcumin

Group II: Cisplatin

In Group I, concurrent cisplatin was given $\left(40 \mathrm{mg} / \mathrm{m}^{2}\right.$, weekly) during the EBRT, with daily dose of oral curcumin (4gm/day). Curcumin is given continuously during the total treatment duration (EBRT as well as ICBT). Group II patients were treated with concurrent cisplatin $\left(40 \mathrm{mg} / \mathrm{m}^{2}\right.$, weekly) alone.

\section{Evaluation}

During treatment, patients were evaluated weekly for acute radiation reactions. The haematological toxicities were assessesd using World Health Organization (WHO) criteria. Acute radiation reactions of skin, mucosa, upper gastrointestinal tract (GIT), lower GIT and genitourinary tract (GUT) were evaluated according to Radiation Therapy Oncology Group (RTOG) criteria. South West Oncology Group (SWOG) criteria was used for evaluating the weight loss. After the treatment was completed, the disease status was evaluated according to WHO criteria. Late toxicities were assessed according to RTOG/ EORTC (European Organization for Research and Treatment of Cancer) late toxicity criteria.

\section{Statistical analysis}

The data thus obtained was analyzed and compared using SPSS software version 20.0. Tumor response rate, radiation toxicities were evaluated between the two groups by the chi-square test. Survival data were calculated by the Kaplan-Meier method and log-rank test to identify any statistical significance between the two groups.

\section{Results}

\section{Patient data}

A total of 60 patients were included in this study. Thereafter, sixty patients were randomized into group I (with curcumin) and group II (without curcumin) as described above. All data are shown in Table 1.
Table I Patient characteristics

\begin{tabular}{|c|c|c|c|}
\hline Characteristics & Group I & Group II & p-value \\
\hline \multicolumn{4}{|l|}{ Age (years) } \\
\hline$\leq 30$ & $3.33 \%$ & $3.33 \%$ & \\
\hline $31-40$ & $16.67 \%$ & $16.67 \%$ & \\
\hline $4 I-50$ & $23.33 \%$ & $26.67 \%$ & 0.844 \\
\hline $51-60$ & $20 \%$ & $23.33 \%$ & \\
\hline $61-70$ & $30 \%$ & $30 \%$ & \\
\hline$>70$ & $6.67 \%$ & $0 \%$ & \\
\hline \multicolumn{4}{|l|}{ Social background } \\
\hline Rural & $66.67 \%$ & $66.67 \%$ & I \\
\hline Urban & $33.33 \%$ & $33.33 \%$ & \\
\hline \multicolumn{4}{|l|}{ KPS } \\
\hline 80 & $90 \%$ & $93.30 \%$ & 0.64 \\
\hline 90 & $10 \%$ & $6.70 \%$ & \\
\hline \multicolumn{4}{|l|}{ Menopausal status } \\
\hline Premenopausal & $30 \%$ & $26.67 \%$ & 0.774 \\
\hline Postmenopausal & $70 \%$ & $73.33 \%$ & \\
\hline \multicolumn{4}{|l|}{ Tumor Morphology } \\
\hline Ulceroproliferative & $90 \%$ & $86.67 \%$ & 0.688 \\
\hline Infiltrative & $10 \%$ & $13.33 \%$ & \\
\hline \multicolumn{4}{|l|}{ Histology } \\
\hline WDSCC & $0 \%$ & $0 \%$ & 0.519 \\
\hline MDSCC & $76.67 \%$ & $83.33 \%$ & \\
\hline PDSCC & $23.33 \%$ & $16.67 \%$ & \\
\hline \multicolumn{4}{|l|}{ Stage } \\
\hline IIB & $60 \%$ & $46.67 \%$ & \\
\hline IIIA & $6.67 \%$ & $13.33 \%$ & 0.67 \\
\hline IIIB & $30 \%$ & $33.33 \%$ & \\
\hline IVA & $3.33 \%$ & $6.67 \%$ & \\
\hline
\end{tabular}

WDSCC, well differentiated squamous cell carcinoma; MDSCC, moderately differentiated squamous cell carcinoma; PDSCC, poorly differentiated squamous cell carcinoma

\section{Treatment received and duration}

All patients in both the groups received the intended dose of 50Gy by external beam radiotherapy. In both the groups, all patients received concomitant cisplatin of $40 \mathrm{mg} / \mathrm{m}^{2}$ weekly. In group I, all patients took $4 \mathrm{gm}$ of curcumin per day during the treatment.

In group I and group II, $63.33 \%$ and $53.33 \%$ of the patients respectively completed the whole treatment within $7-8$ weeks. In group I and group II, $30 \%$ and $33.33 \%$ of the patients took $8-9$ weeks to complete the treatment respectively. In group I, treatment was interrupted for more than one week in two patients (6.67\%). In group II, treatment interruption was seen in four patients $(13.33 \%)$. 


\section{Response of the tumor}

After the completion of treatment, complete tumor response was seen in $70 \%$ of the patients in group I and $66.67 \%$ patients in group II at the end of treatment. In stage IIB, complete response was observed in $83.33 \%$ and $64.29 \%$ of patients in group I and group II respectively. In group I, complete response in stage IIIA and stage IIIB were $100 \%$ and $44.44 \%$ respectively. In group 2 , complete response in stage IIIA and stage IIIB were $75 \%$ and $70 \%$ respectively. In group I and II, complete response in stage IVA was seen in $0 \%$ and $50 \%$ respectively. Overall, partial response was seen in $30 \%$ of the patients in each group. One patient in group II has no response with the treatment. The observations in terms of tumor response were not statistically significant.

\section{Survival rates}

With the median follow up of 24 months, overall survival and disease free survival rates were $93.3 \%, 76.7 \%$ in group I and $90 \%$, $66.7 \%$ in group II respectively. The survival rates of the two groups were not statistically significant. These are represented in Figure 1.

Residual disease occurred in $20 \%$ and $26.67 \%$ of the patients in group I and group II respectively. Recurrence was seen in $3.33 \%$ and $6.67 \%$ of the patients in group I and group II respectively. The distant metastasis to lung was seen in two $(6.67 \%)$ patients and to supraclavicular node was seen in one $(3.33 \%)$ patient; however, these patients did not show any evidence of local disease. Though, findings were not significantly different but they are indicative of slightly better response in group I.

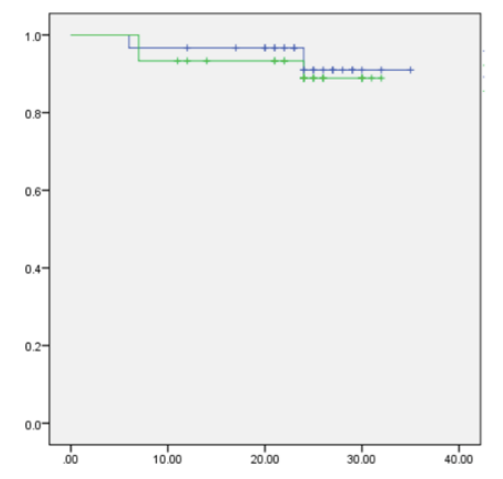

a) Overall Survival rate $(p=0.733)$

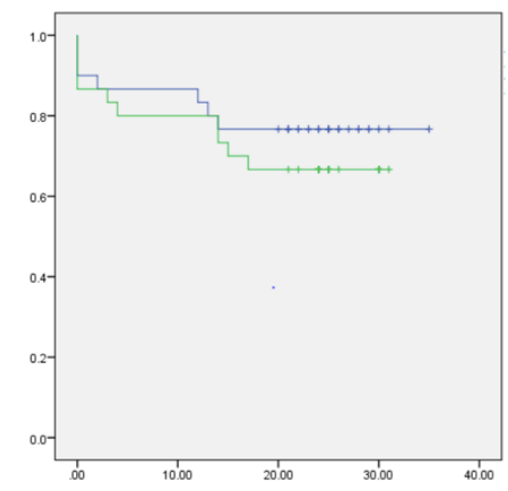

b) Disease Free Survival rate $(p=0.431)$

Figure I Kaplan- Meier curve showing a) Overall survival rate and b) Disease free survival rate of group I (blue line) versus group II (green line).

\section{Toxicity profiles}

Acute radiation toxicity: Grade 2 hematological toxicity $(\mathrm{Hb})$ was seen in $13.33 \%$ and $20 \%$ of the patients in group I and group II respectively. Grade 3 and grade 4 toxicity was not observed in any of the patient. TLC data revealed grade 1 toxicity in $6.7 \%$ and $10 \%$ of the patients in group I and II respectively.

Grade 1 skin reactions were seen in $76.67 \%$ and $33.33 \%$ of the patients in group I and group II respectively. Grade 2 skin reactions were seen in $20 \%$ and $46.67 \%$ of the patients in group I and group II respectively. Grade 3 skin reactions were seen in $3.33 \%$ and $20 \%$ of the patients in group I and group II respectively. The significant difference was found in occurrence of grade 2 and grade 3 skin reactions in the two groups. Acute mucosal toxicity of grade 1 was seen in $63.4 \%$ and $33.3 \%$ of the patients in group I and group II respectively. Acute mucosal toxicity of grade 2 was seen in $23.3 \%$ and $50 \%$ of patients in group I and II respectively, which was significant statistically. Grade 3 mucosal toxicity was seen in $13.3 \%$ and $16.7 \%$ of patients in group I and group II respectively.

Four patients in group I and two patients in group II developed grade 3 upper GI toxicity. Grade 4 toxicity was not observed in any group. Lower GI grade 3 toxicity was observed in one patient each in group I and group II. Grade 1 genitourinary toxicity was observed in seven patients in group I and nine patients in group II. Grade 2, 3 and 4 toxicity was not observed in any of the groups. The findings were not significant. In both the groups, no significant weight loss was found. All data is shown in Table 2.

Table 2 Acute radiation toxicity of group I versus group II

\begin{tabular}{llll}
\hline Acute toxicities & Group I & Group II & p- value \\
\hline Skin toxicity & & & \\
Grade 2 & $20 \%$ & $46.70 \%$ & 0.028 \\
Grade 3 & $3.30 \%$ & $20 \%$ & 0.044 \\
Mucosal toxicity & & & \\
Grade 2 & $23.30 \%$ & $50 \%$ & 0.032 \\
Grade 3 & $13.30 \%$ & $16.70 \%$ & 0.717 \\
Upper GIT toxicities & & & \\
Grade I & $20 \%$ & $26.70 \%$ & 0.54 \\
Grade 2-3 & $53.30 \%$ & $43.30 \%$ & 0.44 \\
Lower GIT toxicities & & & \\
Grade I & $86.70 \%$ & $83.30 \%$ & 0.72 \\
Grade2-3 & $13.30 \%$ & $16.70 \%$ & \\
GUT toxicities & & & \\
Grade I & & $30 \%$ & 0.56 \\
Grade 2 & $77 \%$ & $70 \%$ & \\
\hline gastroints & $23 \%$ & &
\end{tabular}

GIT, gastrointestinal tract; GUT, genitourinary tract

\section{Late radiation toxicity}

Late cutaneous grade 1 toxicity was seen in $70 \%$ and $80 \%$ of patients in group I and II respectively. Grade 2 cutaneous toxicity was observed in $13.33 \%$ and $10 \%$ of patients in group I and II respectively. The observations were not statistically significant. Late subcutaneous grade 2 toxicity was seen in $16.67 \%$ and $23.33 \%$ of patients in group I and II respectively. The observations were not statistically significant. 
Late mucosal grade 1 toxicity was seen in $20 \%$ and $23.33 \%$ patients of the group I and II respectively. Late mucosal grade 2 toxicity was seen in group II in $3.33 \%$ of patients. The observations were not statistically significant.

Grade 2 toxicity of lower GI was observed in one patient of group II and not seen in group I. Grade 1 toxicity was seen in $16.67 \%$ and $23.33 \%$ of patients in group I and group II respectively. Grade 1 bladder toxicity was observed in $7 \%$ and $10 \%$ of patients in group and II respectively. The observations were not statistically significant. All observations are shown in Table 3.

Table 3 Late radiation toxicity of group I versus group II

\begin{tabular}{llll}
\hline Late toxicities & Group I & Group II & p- value \\
\hline Cutaneous & & & \\
Grade 0 & $16.67 \%$ & $10 \%$ & 0.843 \\
Grade I & $70 \%$ & $80 \%$ & \\
Grade 2 & $13.33 \%$ & $10 \%$ & \\
Subcutaneous & & & \\
Grade 0 & $23.33 \%$ & $23.33 \%$ & \\
Grade I & $60 \%$ & $54 \%$ & \\
Grade 2 & $16.67 \%$ & $23.33 \%$ & \\
Mucosal & & & 0.559 \\
Grade 0 & $80 \%$ & $73.33 \%$ & \\
Grade I & $20 \%$ & $23.33 \%$ & \\
Lower GIT & & & \\
Grade 0 & $83.33 \%$ & $73.33 \%$ & \\
Grade I & $16.67 \%$ & $23.33 \%$ & \\
Grade 2 & $0 \%$ & $3.33 \%$ & \\
Bladder & & & \\
Grade 0 & & & \\
Grade I & & & \\
\hline & & & \\
\hline
\end{tabular}

\section{Discussion}

EBRT followed by ICBT was the best option till 1990s, thereafter the treatment paradigm has shifted to concomitant chemoradiation with cisplatin based chemotherapy. Five-year OS of patients treated with chemoradiation now approach $70 \%$ to $80 \%$ for stage IIB disease, $50 \%$ to $60 \%$ for stage IIIB disease, and perhaps $15 \%$ to $25 \%$ for stage IVA disease. ${ }^{2-7}$

This study was conducted to observe the effect of curcumin in locally advanced cervical cancer, when given with concomitant chemoradiation. This is the first study in the published literature trying to evaluate the role of curcumin in cervical cancer patients.

It has been revealed that curcumin sensitizes tumors to different chemotherapeutic agents including paclitaxel, vincristine, doxorubicin, 5-FU, cisplatin and gemcitabine. Chemosensitization has been observed in cancers such as breast, colon, pancreas, cervix, head and neck and in multiple myeloma, leukemia and lymphoma. ${ }^{9}$ Curcumin is a highly pleiotropic molecule that modulates numerous targets, including cell cycle (cyclin D1 and cyclin E), apoptosis (activation of caspases and down-regulation of antiapoptotic gene products), proliferation (Human epidermal growth factor receptor-2, Epidermal growth factor receptor, and Activating protein-1), survival (Phosphatidyl inositol-3-kinase/ protein kinase B pathway), invasion (Matrix metalloproteinase-9 and adhesion molecules), angiogenesis (Vascular endothelial growth factor), metastasis (Chemokine receptor type 4) and inflammation (Nuclear factor - kappa B, Tumor Necrosis Factor, Interleukin-6, Interleukin-1, Cyclooxygenase-2 and 5-Lipoxygenase) [10]. Curcumin has been shown to inhibit cell proliferation, cell cycle arrest and stimulate apoptosis via modulation of other transcription factors, such as AP-1, Erg-1, p53, $\beta$-catenin, Notch-1, Hif-1, and PPAR- $\alpha .{ }^{11}$

Transcription of pathogenic HPVs during keratinocyte differentiation and thus, progression of cervical cancer can be controlled by curcumin as shown by Prusty et al., ${ }^{12}$ in a study on HeLa cells. ${ }^{12}$ Qiao et al., ${ }^{13}$ has shown that curcumin enhanced the response of non hodgkin lymphoma cells to ionizing radiation. It has been observed in the study that the role of curcumin in inducing $\mathrm{G} 2 / \mathrm{M}$ phase arrest and inhibiting the mTOR-NF- $\mathrm{kB}$ pathway is responsible for the effect. This has offered great potential for curcumin to be used in conjunction with radiotherapy for NHL in order to increase the efficiency of the treatment. ${ }^{13}$ It was recently demonstrated by Javvadi et al., ${ }^{14}$ that pretreatment of two cervical cancer cell lines $\mathrm{HeLa}$ and $\mathrm{SiHa}$ with curcumin prior to ionizing radiation resulted in radiosensitization of cancer cells. ${ }^{14}$ Khafif et al., ${ }^{15}$ investigated that curcumin can sensitize squamous cell carcinoma cells to the ionizing effects of irradiation. A significant decrease in the ability to form colonies in vitro following pretreatment with curcumin was found at all radiation doses establishing that curcumin may serve as an adjuvant in radiotherapy. ${ }^{15}$

Curcumin has also shown the potential role in the prevention and treatment of colorectal cancer in combination with other agents. The combination of curcumin and quercetin has shown to decrease the number and size of polyps after 6 months of treatment without any appreciable toxicity. ${ }^{16}$ Golombick et al. ${ }^{17}$ has done a study to determine the effects of curcumin on plasma cells and osteoclasts in patients with Monoclonal gammopathy of undetermined significance (MGUS). This study enrolled twenty-six patients with MGUS, who were randomly assigned to two groups. In group 1, 17 patients were given curcumin at the start of study and were then crossed over to placebo after 3 months. In group 2, nine patients were given placebo initially and then crossed over to curcumin. Paraprotein load was found to be decreased in the ten patients. Out of these ten patients, five had a $12 \%$ to $30 \%$ reduction in paraprotein levels while receiving curcumin therapy. Also, $>25 \%$ decrease in urinary $N$-telopeptide of type I collagen was noticed in $27 \%$ of patients receiving curcumin. The study suggested the therapeutic importance of curcumin against MGUS. ${ }^{17}$

In our study on locally advanced cervical cancer, the results of concomitant chemoradiation with or without curcumin were comparable. Though, slightly better response was seen in group I in terms of tumor response, disease free survival and overall survival, but no statistically significant difference was observed between the two groups. In group I, the treatment was interrupted in two patients $(6.67 \%)$ during the external beam radiotherapy. These patients had severe acute skin radiation reactions. These patients complete their dose of external beam radiotherapy in more than six weeks. In group II, treatment duration was increased in four patients $(13.33 \%)$. Grade 3 skin and mucosal reactions were seen in all of these patients 
resulting in the delay of the treatment. These four patients took more than six weeks to complete the planned treatment. In group I and group II, $6.67 \%$ and $13.33 \%$ of the patients respectively completed the whole treatment in more than 9 weeks due to associated severe acute radiation toxicity. The observations regarding the total treatment duration were similar to the study by Eifel et al. ${ }^{6}$

Perez et al., ${ }^{18}$ noted a major impact of prolongation of treatment time on pelvic tumor control in stages IB, IIA, and IIB. In stage III, although the rate of pelvic failure was higher with prolongation of treatment time, the difference was not statistically significant. ${ }^{18}$ In our study, no significant association was found in the tumor response or disease status with prolongation of time.

In a study by Maduro et al., ${ }^{19}$ acute and long-term toxicity of radiotherapy for cervical cancer were assessed. Acute toxicity of radiotherapy was reported in $61 \%$ of the patients in the rectosigmoid, in $27 \%$ as urological, in $27 \%$ as skin and in $20 \%$ as gynaecological toxicity. ${ }^{19}$ The acute toxicities of GIT and GUT didn't differ much in the two groups. There was insignificant increase in the upper GI toxicity, especially nausea and vomiting in group I. Our study has shown the significant benefit in the acute skin radiation toxicity and acute mucosal radiation toxicity in the group taking curcumin. The results in our study are consistent with the results observed by Ryan et al.,$^{20}$ in their study. They found out that the oral curcumin daily during radiotherapy, reduced the severity of radiation dermatitis in breast cancer patients. ${ }^{20}$ The arm containing curcumin had fewer moist desquamation and also reduced Radiation Dermatitis Severity (RDS) score at end of the treatment compared to placebo. ${ }^{20}$ In the present study, the two groups didn't differ significantly in terms of late radiation toxicity.

\section{Conclusion}

The present study revealed statistically significant improvements in the radiation induced skin and mucosal toxicity with the addition of curcumin and, therefore, lesser treatment delay while giving radiotherapy. The limitation of the study included the small sample size. Taking into account the comparable response rate, comparable treatment related toxicities, significantly less radiation induced skin and mucosal toxicity with curcumin, the study is suggestive that the future clinical trials should consider to investigate the potential effectiveness of curcumin in the treatment of locally advanced uterine cancer.

\section{Acknowledgements}

None.

\section{Conflict of interest}

The author declares no conflicts of interest.

\section{Funding}

No sources of funding were received.

\section{References}

1. Ferlay J, Soerjomataram I, Ervik M, et al. Cancer Incidence and Mortality Worldwide: IARC Cancer Base No. 11. Lyon, France: International Agency for Research on Cancer; 2012.

2. Rose PG, Bundy BN, Watkins EB, et al. Concurrent cisplatin based radiotherapy and chemotherapy for locally advanced cervical cancer. $N$ Engl J Med. 1999;340(15):1144-1153.
3. Whitney CW, Sause W, Bundy BN, et al. Randomized comparison of fluorouracil plus cisplatin versus hydroxyurea as an adjunct to radiation therapy in stage IIB-IVA carcinoma of the cervix with negative paraaortic lymph nodes: a Gynecologic Oncology Group and Southwest Oncology Group study. J Clin Oncol. 1999;17(5):1339-1348.

4. Peters WA III, Liu PY, Barrett RJ, et al. Concurrent chemotherapy and pelvic radiation therapy compared with pelvic radiation therapy alone as adjuvant therapy after radical surgery in high-risk early-stage cancer of the cervix. J Clin Oncol. 2000;18(8):1606-1613.

5. Keys H, Bundy B, Stehman F, et al. Cisplatin, radiation, and adjuvant hysterectomy compared with radiation and adjuvant hysterectomy for bulky stage IB cervical carcinoma. N Engl J Med. 1999;340(15):11541161.

6. Eifel PJ, Winter K, Morris M, et al. Pelvic irradiation with concurrent chemotherapy versus pelvic and para-aortic irradiation for high risk cervical cancer: an update of radiation therapy oncology group trial (RTOG) 90-01. J Clin Oncol. 2004;22(5):872-880.

7. Morris M, Eifel PJ, Lu J, et al. Pelvic radiation with concurrent chemotherapy versus pelvic and para-aortic radiation for high-risk cervical cancer. A randomized Radiation Therapy Oncology Group clinical trial. N Engl J Med. 1999;340(15):1137-1143.

8. Goel A, Aggarwal BB. Curcumin, the Golden Spice From Indian Saffron, Is a Chemosensitizer and Radiosensitizer for Tumors and Chemoprotector and Radioprotector for Normal Organs. Nutr cancer. 2010;62(7):919-930.

9. Venkatraman M, Anto RJ, Nair A, et al. Biological and chemical inhibitors of NF-kappaB sensitize SiHa cells to cisplatin-induced apoptosis. Mol Carcinog. 2005;44(1):51-59.

10. Anand P, Sundaram C, Jhurani S, et al. Curcumin and cancer: An old-age disease with an age-old solution. Cancer Lett. 2008; 267(1):133-164.

11. Vallianou N, Evangelopoulos A, Schizas N, et al. Potential anticancer properties and mechanisms of action of curcumin. Anticancer Res. 2015;35(2):645-652.

12. Prusty BK, Das BC. Constitutive activation of transcription factor AP-1 in cervical cancer and suppression of human papillomavirus (HPV) transcription and AP-1 activity in HeLa cells by curcumin. Int $J$ Cancer. 2005;113(6):951-960.

13. Qiao Q, Jiang Y, Li G. Curcumin enhances the response of non-Hodgkin's lymphoma cells to ionizing radiation through further induction of cell cycle arrest at the G2/M phase and inhibition of mTOR phosphorylation. Oncol Rep. 2013;29(1):380-386.

14. Javvadi P, Segan AT, Tuttle SW, et al. The chemopreventive agent curcumin is a potent radiosensitizer of human cervical tumor cells via increased reactive oxygen species production and overactivation of the mitogenactivated protein kinase pathway. Mol Pharmacol. 2008;73(5):1491-1501.

15. Khafif A, Hurst R, Kyker K, et al. Curcumin: a new radio-sensitizer of squamous cell carcinoma cells. Otolaryngol Head Neck Surg. 2005;132(2):317-321.

16. Cruz-Correa M, Shoskes DA, Sanchez P, et al. Combination treatment with curcumin and quercetin of adenomas in familial adenomatous polyposis. Clin Gastroenterol Hepatol. 2006;4(8):1035-1038.

17. Golombick T, Diamond TH, Badmaev V, et al. The potential role of curcumin in patients with monoclonal gammopathy of undefined significance-its effect on paraproteinemia and the urinary N-telopeptide of type I collagen bone turnover marker. Clin Cancer Res. 2009;15(18):5917-5922.

18. Perez CA, Grigsby PW, Castro-Vita H, et al. Carcinoma of the uterine cervix: I. Impact of prolongation of treatment time and timing of brachytherapy on outcome of radiation therapy. Int J Radiat Oncol Biol Phys. 1995;32(5):1275-1288. 
19. Maduro JH, Pras E, Willemse PH, et al. Acute and long-term toxicity following radiotherapy alone or in combination with chemotherapy for locally advanced cervical cancer. Cancer Treat Rev. 2003;29(6):471488.
20. Ryan JL, Heckler CE, Ling M, et al. Curcumin for radiation dermatitis: a randomized, double-blind, placebo-controlled clinical trial of thirty breast cancer patients. Radiat Res. 2013;180(1):34-43. 